\title{
Shingles and Pericarditis: A Rare Combination
}

\author{
Rakan M. Nasreddine1, Chirine A. Mollaei², Joudy N. Bahous², Eid E. Azar1', Claude M. Afif ${ }^{*}$ \\ ${ }^{1}$ Division of Infectious Disease, St. George Hospital University Medical Center, in association with the University \\ of Balamand, Beirut, Lebanon \\ ${ }^{2}$ Division of Pulmonary and Critical Care Medicine, St. George Hospital University Medical Center, in association \\ with the University of Balamand, Beirut, Lebanon \\ Email: ${ }^{*}$ claude.afif@balamand.edu.lb
}

Received 8 April 2015; accepted 17 May 2015; published 20 May 2015

Copyright (C) 2015 by authors and Scientific Research Publishing Inc.

This work is licensed under the Creative Commons Attribution International License (CC BY).

http://creativecommons.org/licenses/by/4.0/

c) (i) Open Access

\begin{abstract}
Clinical infection with varicella in both its' forms, primary and reactivation, can be associated with a variety of complications. Cardiac complications, though very rare, have been associated with the primary form of varicella zoster and as such should be recognized in order to initiate early treatment and prevent morbidity and mortality. However, cardiac complications have not been described in association with the reactivation form of varicella. We report a case of an adult immunocompetent male who presented with herpes zoster complicated by pericarditis with pericardial effusion and a positive varicella zoster virus (VZV) polymerase chain reaction (PCR) in pericardial fluid.
\end{abstract}

\section{Keywords}

\section{Herpes Zoster, Shingles, Pericarditis}

\section{Introduction}

Varicella zoster virus (VZV) is one of eight herpesviruses known to cause human infection and is distributed worldwide. VZV infection causes two clinically distinct forms of disease. Primary VZV infection results in the diffuse vesicular rash of varicella, also known as chickenpox. Endogenous reactivation of latent VZV typically results in a localized skin infection known as herpes zoster, or shingles [1]. Infection can sometimes be associated with a variety of complications that are more common in adults and in immunocompromised patients [1] [2]. Complications involving the cardiovascular system are rare however, specifically in the shingles form.

"Corresponding author. 


\section{Case Presentation}

An 87-year-old man presented for fever, fatigue, cough and dyspnea upon exertion. Five days earlier, he was diagnosed with shingles of the right neck (C2 territory) for which he was prescribed valacyclovir. The patient is known to have atrial fibrillation and atrio-ventricular block for which a pacemaker was inserted seven years earlier. Upon admission, the patient looked tired; however his physical examination did not reveal any abnormalities except for crusting lesions over the neck. A chest X-ray on admission revealed an enlarged heart obscuring the left lung base (Figure 1(a)). CT scan of the chest showed interstitial infiltrates with ground glass appearance in the left upper lobe, lingula and both bases along with a moderate to large pericardial effusion (Figure 1(b)). An echocardiogram revealed normal contractility with no cardiomyopathy, with an estimated 1 liter of pericardial effusion without signs of tamponade. CT guided pericardiocentesis drained $500 \mathrm{ml}$ of a non-hemorrhagic exudative fluid, the analysis of which showed a lactate dehyrdogenase level of $189 \mathrm{U} / \mathrm{L}$ with an effusion/serum ratio of 0.99, a total protein level of $4.6 \mathrm{~g} / \mathrm{dL}$ with an effusion/serum ratio of 0.68 , a glucose level of $136 \mathrm{mg} / \mathrm{dL}$, a white blood cell count of $100 / \mathrm{mm}^{3}$ with a lymphocytic predominance of $98 \%$, and a red blood cell count of $3000 / \mathrm{mm}^{3}$. Microbiological evaluation for tuberculosis and rheumatic work-up was non-revealing. However, further analysis of the pericardial fluid using the DNA microarray technique for the detection of gene expression in cells revealed both VZV and Enterovirus.

The patient was treated with Colchicine (1 mg daily) and valacyclovir was continued to complete a three week course. On discharge, patient showed significant improvement in dyspnea and overall well-being while repeat echocardiography and chest X-ray showed almost complete resolution. Follow up at 3 months showed complete clinical and radiological resolution of signs and symptoms.

\section{Discussion}

Herpes zoster, also known as shingles, results from reactivation of endogenous latent VZV infection within the sensory ganglia. Clinically, shingles is characterized initially by pain or discomfort in the involved dermatome, usually without constitutional symptoms. Local edema and erythema then appear followed by the development of a maculopapular and vesicular rash which eventually evolves into crusts. Vesicular lesions that follow a dermatomal pattern should alert the clinician for a diagnosis of shingles. Viral cultures are currently the best method for establishing the diagnosis. Scraping the base of a vesicle and performing a Tzanck smear on the sample may demonstrate multinucleated giant cells which raises the suspicion for the diagnosis; however this test does not distinguish HSV from VZV. PCR of the vesicular lesions is becoming more and more the diagnostic test of choice because of sensitivity, specificity, and specimen stability.

The most common complication associated with herpes zoster is post-herpetic neuralgia. Less frequent complications include ocular complications (keratitis, iridocyclitis, secondary glaucoma, and loss of sight), neuro-

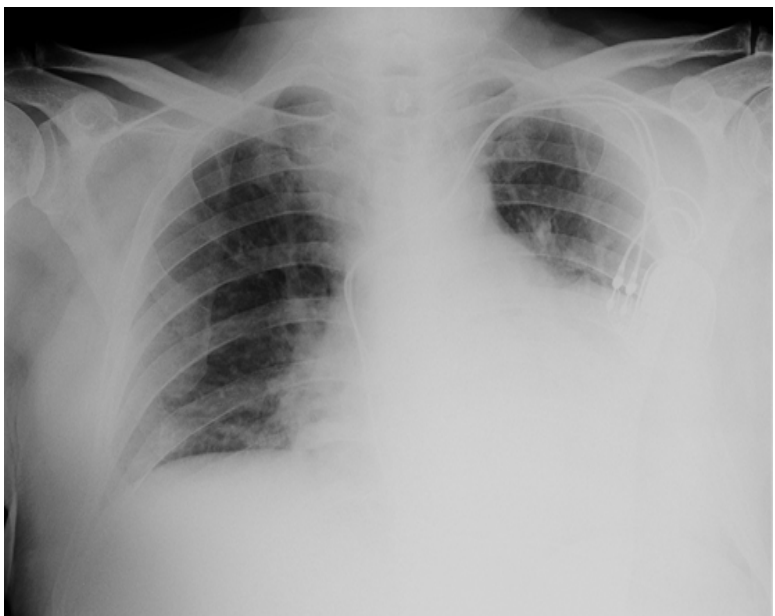

(a)

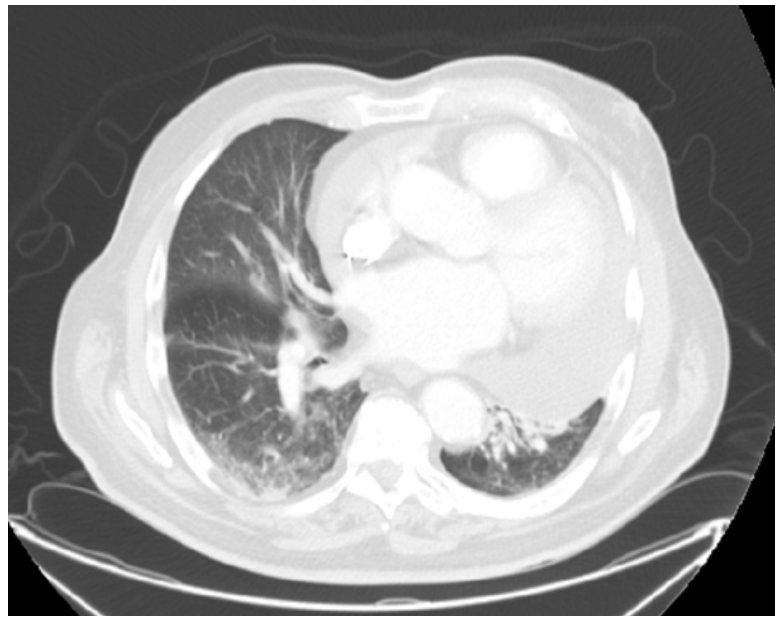

(b)

Figure 1. (a) Chest X-ray showing cardiomegaly with silhouetting of left diaphragm; (b) Chest CT (parenchymal view): interstitial infiltrates with ground glass appearance along with a moderate to large pericardial effusion. 
logical complications (various motor neuropathies, meningitis, encephalitis, and Guillain-Barre syndrome), secondary bacterial infection of vesicles, and otologic complications (Ramsay Hunt syndrome). Immunocompromised patients are at an increased risk of developing complicated herpes zoster infections including cutaneous dissemination and visceral end organ involvement including pneumonia, hepatitis, and encephalitis.

Cardiac complications however, are extremely rare. An extensive search of the PUBMED, EMBASE, and The Cochrane Library databases using the search terms "herpes zoster", "varicella zoster", "pericarditis", and "vzv"; either alone or in combination was performed. Articles published in English were reviewed. Myocardial and pericardial complications associated with primary VZV infection have been described. A review of these reports revealed varying complications with morbidity and mortality ranging from full recovery to death [3]-[11].

However, to the best of our knowledge, this is the first report of pericarditis associated with herpes zoster. As the virus is difficult to document in tissues in association with VZV-associated complications, the recognition of the varied spectrum of disease caused by VZV is always a challenge. In such instances, viral PCR detection proves helpful to confirm the diagnosis. As such, this report should serve to shed light on the potential for cardiac complications associated with herpes zoster.

There is limited data concerning the use of antiviral agents (acyclovir), intravenous immunoglobulin (IVIG), and supportive treatments when managing patients with complications of VZV, especially cardiac complications. Reviewing the literature, there are no clear guidelines for the management of patients with myopericarditis either associated with the primary or reactivation forms of VZV. Indeed, according to Cohen, it has been shown that the early initiation of antiviral therapy for herpes zoster hastens the resolution of lesions, reduces the formation of new lesions, reduces viral shedding, and decreases the severity of acute pain [1]. In addition, as described by the review article of Mohsen et al., on the rare occasion in which an immunocompetent adult develops a complication of varicella, such as pneumonia, the benefit is there for the use of intravenous acyclovir [12]. In immunocompromised patients however, the risk of cutaneous and visceral dissemination of VZV is well recognized, and these include encephalitis, pneumonia, and hepatitis [1] [13]. In Gnann's review article, the use of high dose intravenous acyclovir therapy resulted in substantially reduced mortality associated with disseminated zoster [13].

In this patient the timely initiation of valacyclovir did not prevent the occurrence of pericarditis. Although there is no standard therapy for VZV pericarditis, we elected to administer valacyclovir for three weeks, considering this complication as visceral involvement. Whether the antiviral treatment contributed to the improvement of the patient or not remains to be answered, since no objective markers can be followed and no repeated pericardiocentesis would have been feasible to evaluate the numeric changes in viral concentration of the studied fluid. Furthermore, the viral PCR was positive for both VZV and Enterovirus, questioning the culprit, knowing that the latter is a known common cause of viral pericarditis. However, the isolation of VZV argues for its' role at least as a co-infection.

\section{Conclusion}

Clinical infection with varicella in both its' forms is most often but not always benign. However, clinicians should be aware of potential cardiovascular presentations and sequelae of shingles, and patients should be investigated further when the suspicion is raised. Finally, the development of clear guidelines with regards to the management of such patients is warranted.

\section{References}

[1] Cohen, J.I. (2013) Herpes Zoster. The New England Journal of Medicine, 369, 255-263. http://dx.doi.org/10.1056/NEJMcp1302674

[2] Fashner, J. and Bell, A.L. (2011) Herpes Zoster and Postherpetic Neuralgia: Prevention and Management. American Family Physician, 83, 1432-1437.

[3] Hackel, D.B. (1953) Myocarditis in Association with Varicella. The American Journal of Pathology, 29, 369-379.

[4] Sampson, C.C. (1959) Varicella Myocarditis. Journal of the National Medical Association, 51, 138-139.

[5] Winfield, C.R. and Joseph, S.P. (1980) Herpes Zoster Pericarditis. British Heart Journal, 43, 597-599. http://dx.doi.org/10.1136/hrt.43.5.597 
[6] Seddon, D.J. (1986) Pericarditis with Pericardial Effusion Complicating Chickenpox. Postgraduate Medical Journal, 62, 1133-1134. http://dx.doi.org/10.1136/pgmj.62.734.1133

[7] Abrams, D., Derrick, G., Penny, D.J., Shinebourne, E.A. and Redington, A.N. (2001) Cardiac Complications in Children Following Infection with Varicella Zoster Virus. Cardiology in the Young, 11, 647-652. http://dx.doi.org/10.1017/S1047951101001019

[8] Nandeesh, B.N., Mahadevan, A., Yasha, T.C. and Shankar, S.K. (2009) Hemorrhagic Pericarditis in a Child with Primary Varicella Infection (Chickenpox). Indian Journal of Pathology \& Microbiology, 52, 237-239. http://dx.doi.org/10.4103/0377-4929.48930

[9] Kao, K.L., Yeh, S.J. and Chen, C.C. (2010) Myopericarditis Associated with Varicella Zoster Virus Infection. Pediatric Cardiology, 31, 703-706. http://dx.doi.org/10.1007/s00246-009-9637-5

[10] Sayin, M.R., Karabag, T., Celik, O., Cetiner, M.A., Cil, C. and Aydin, M. (2011) Varicella Zoster Myopericarditis. Journal of Medical Cases, 2, 287-288. http://dx.doi.org/10.4021/jmc398w

[11] De, A., Myridakis, D., Kerrigan, M. and Kiblawi, F. (2011) Varicella Myopericarditis Mimicking Myocardial Infarction in a 17-Year-Old Boy. Texas Heart Institute Journal, 38, 288-290.

[12] Mohsen, A.H. and McKendrick, M. (2003) Varicella Pneumonia in Adults. European Respiratory Journal, 21, 886891. http://dx.doi.org/10.1183/09031936.03.00103202

[13] Gnann, J.W. (2002) Varicella-Zoster Virus: Atypical Presentations and Unusual Complications. The Journal of Infectious Diseases, 186, S91-S98. http://dx.doi.org/10.1086/342963 